\title{
Accurate calculation of the eigenvalues of a new simple class of superpotentials in SUSY quantum mechanics
}

Francisco M. Fernández*

INIFTA (UNLP, CCT La Plata-CONICET), División Química Teórica,

Blvd. 113 S/N, Sucursal 4, Casilla de Correo 16,

1900 La Plata, Argentina

Received 04 July 2012; accepted 17 February 2013

\begin{abstract}
:
We obtain accurate eigenvalues for two recently derived SUSY partner Hamiltonians. We improve the Rayleigh-Ritz variational method proposed by the authors and show how to apply the Riccati-Padé method to those particular partner potentials.

PACS (2008): 03.65.Ge

Keywords:

SUSY partner Hamiltonians • variational calculation • Riccati-Padé method • accurate eigenvalues

(c) Versita sp. z o.o.
\end{abstract}

\section{Introduction}

In a recent paper Marques et al. [1] derived two new simple supersymmetric partner potentials and showed that the ground-state eigenfunction of one of the Hamiltonian operators can be obtained exactly. In order to calculate the remaining states they resorted to the Rayleigh-Ritz variational method. However, the convergence rate of their approach appears to be insufficient to reveal the relation between the eigenvalues of the partner Hamiltonians clearly.

The authors also estimated the ground-state energy of the quartic anharmonic oscillator by means of perturbation

*E-mail: fernande@quimica.unlp.edu.ar theory starting from the known solution for one of the partner Hamiltonian operators.

The purpose of this paper is twofold. First we improve the variational method proposed by Marques et al. [1] and, second, we show that the Riccati-Padé method (RPM) [24] provides highly accurate results for the eigenvalues of those partner Hamiltonian operators. In addition, we show that exactly the same Rayleigh-Ritz variational method applied to the SUSY partner Hamiltonians is also suitable for the calculation of the eigenvalues of the quartic anharmonic oscillator.

In Section 2 we apply the Rayleigh-Ritz variational method and the RPM to the pair of SUSY Hamiltonians proposed by Marques et al. [1] and also to the quartic oscillator. In Section 3 we comment on those approaches and draw conclusions. 


\section{SUSY partners}

Table 1. Eigenvalues $E_{n}^{-}$from the Rayleigh-Ritz method.

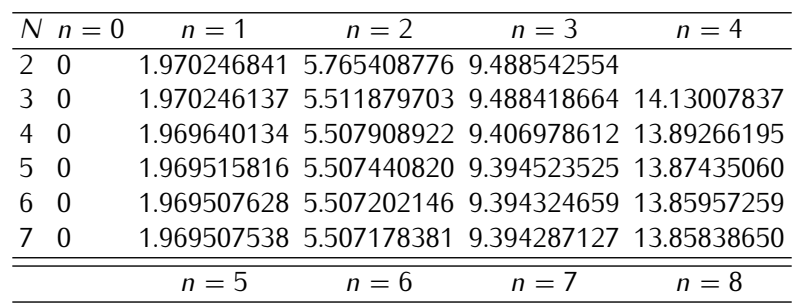

\begin{tabular}{llllll}
\hline 2 & & & & \\
3 & 19.48962926 & & & \\
4 & 19.02107688 & 24.09989393 & 33.11107172 & \\
5 & & 18.66009505 & 24.07406802 & 29.81927599 & 35.91298387 \\
6 & 18.64895855 & 23.84777162 & 29.26028194 & 35.65656448 \\
7 & & 18.64704858 & 23.80790714 & 29.25748454 & 34.98577701 \\
\hline
\end{tabular}

Table 2. Eigenvalues $E_{n}^{+}$from the Rayleigh-Ritz method.

\begin{tabular}{lcccc}
\hline$N$ & $n=0$ & $n=1$ & $n=2$ & $n=3$ \\
\hline 2 & 1.970246841 & 5.529040685 & 9.488542554 & 14.35721210 \\
3 & 1.970246137 & 5.514418950 & 9.488418664 & 14.00103534 \\
4 & 1.969640134 & 5.510107538 & 9.406978612 & 13.92544875 \\
5 & 1.969515816 & 5.507493533 & 9.394523525 & 13.86389218 \\
6 & 1.969507628 & 5.507185747 & 9.394324659 & 13.85851252 \\
7 & 1.969507538 & 5.507178915 & 9.394287127 & 13.85851126 \\
\hline \hline \multicolumn{5}{c}{$n=4$} \\
\hline 2 & $n=5$ & $n=6$ & $n=7$ \\
3 & 19.48962926 & 24.52838046 & & \\
4 & 19.02107688 & 24.52809061 & 33.11107172 & 37.63335229 \\
5 & 18.66009505 & 23.91353748 & 29.81927599 & 36.33167441 \\
6 & 18.64895855 & 23.81074205 & 29.26028194 & 35.02577244 \\
7 & 18.64704858 & 23.81074194 & 29.25748454 & 34.98424967 \\
\hline
\end{tabular}

The partner Hamiltonian operators are given by [1]:

$$
H_{ \pm}=-\frac{d^{2}}{d x^{2}}+x^{4} \pm 2|x|
$$

where we have chosen $g=1$ without loss of generality. In order to solve the Schrödinger equation

$$
H_{ \pm} \psi_{n}^{ \pm}=E_{n}^{ \pm} \psi_{n}^{ \pm}
$$

approximately, Marques et al. [1] chose the nonorthogonal basis set

$$
\varphi_{j}(x)=x^{j-1} e^{-|x|^{3} / 3}, j=1,2, \ldots
$$

Table 3. Eigenvalues for the partner SUSY Hamiltonian operators from the Riccati-Padé method.

\begin{tabular}{lc}
\hline \multicolumn{1}{c}{ Even $(s=0)$} & \multicolumn{1}{c}{ Odd $(s=1)$} \\
\hline \hline 0 & 1.9695075137502948249 \\
1.9695075137502948249 & 5.5071777771459699676 \\
5.5071777771459699676 & 9.3942674378738914658 \\
9.3942674378738914658 & 13.858371936541300147 \\
13.858371936541300147 & 18.645975633988444799 \\
18.645975633988444799 & 23.807185917985766918 \\
23.8071859179857669 & 29.2325545506214961 \\
29.23255455062149608 & 34.9463357188906106 \\
34.94633571889061 & \\
\hline
\end{tabular}

and derived a single secular equation for both the even and odd states. Since states of different parity do not mix the dimension of their secular equation is twice the size of what would be actually necessary for a calculation of the same accuracy.

In this paper we propose to work on the half line $x \in$ $[0, \infty)$ taking into account the appropriate boundary conditions at the origin: $\psi(0) \neq 0, \psi^{\prime}(0)=0$ for the even states and $\psi(0)=0, \psi^{\prime}(0) \neq 0$ for the odd ones. The appropriate basis sets are

$$
\begin{aligned}
& \varphi_{j}^{e}=x^{j} e^{-x^{3} / 3}, j=0,2,3, \ldots, \\
& \varphi_{j}^{o}=x^{j} e^{-x^{3} / 3}, j=1,2,3, \ldots,
\end{aligned}
$$

for the former and latter case, respectively.

Tables 1 and 2 show Rayleigh-Ritz results for the first eigenvalues of $H_{-}$and $H_{+}$, respectively, in terms of the size $N$ of the basis sets. We appreciate that the variational results decrease with the number of basis functions as expected. However, we are not aware of any proof that either of the basis sets (3) or (4) is complete. We see that present results are smaller, and therefore more accurate, than those of Marques et al. [1]. In addition, the results in their tables 1 and 2 exhibit unchanged entries when the size of the basis set increases from $m$ to $m+1$ which reflects the fact that the added basis function improves the even (odd) state but has no effect on the odd (even) one. As a result their matrices are twice the size of what is necessary for a calculation of the same accuracy.

The results of Tables 1 and 2, as well as those of Marques et al. [1], suggest that $E_{n}^{-}=E_{n-1}^{+}, n=1,2, \ldots$, as expected for a SUSY pair of partner Hamiltonian operators. However, those variational results are not sufficiently accurate and, as argued above, we are not aware of any proof of convergence. In order to obtain more accurate results we resort to the RPM [2-4]. To this end we consider 
Table 4. Eigenvalues $E_{n}$ for the quartic oscillator.

\begin{tabular}{|c|c|c|c|}
\hline$N$ & $n=0$ & $n=1$ & $n=2$ \\
\hline 2 & 1.077335422 & 3.804924324 & 8.102531212 \\
\hline 3 & 1.061889825 & 3.802939362 & 7.486487866 \\
\hline 4 & 1.060417725 & 3.800572274 & 7.456738018 \\
\hline 5 & 1.060362727 & 3.799746874 & 7.456540880 \\
\hline 6 & 1.060362727 & 3.799674368 & 7.455826496 \\
\hline 7 & 1.060362223 & 3.799673299 & 7.455703721 \\
\hline RPM & 1.0603620904841828996 & 3.7996730298013941688 & 7.4556979379867383922 \\
\hline & $n=3$ & $n=4$ & $n=5$ \\
\hline 2 & 11.86759677 & & \\
\hline 3 & 11.77079816 & 16.91718138 & 21.92232689 \\
\hline 4 & 11.67894913 & 16.29307103 & 21.79883488 \\
\hline 5 & 11.64634787 & 16.29029658 & 21.28503933 \\
\hline 6 & 11.64483615 & 16.26577116 & 21.24138553 \\
\hline 7 & 11.64480669 & 16.26191320 & 21.24054440 \\
\hline RPM & 11.644745511378162021 & 16.261826018850225938 & 21.238372918235940024 \\
\hline
\end{tabular}

the modified logarithmic derivative of the wavefunction $\psi$

$$
f(x)=\frac{s}{x}-\frac{\psi^{\prime}(x)}{\psi(x)}
$$

where $s=0$ or $s=1$ for even or odd states, respectively. It satisfies the Riccati equation

$$
f^{\prime}(x)=f(x)^{2}-\frac{2 s}{x} f(x)+E-x^{4} \mp 2 x, x>0
$$

and can be expanded in a Taylor series about the origin

$$
f(x)=\sum_{j=0}^{\infty} f_{j} x^{j}
$$

where the coefficients $f_{j}$ can be easily obtained from the Riccati equation (6). We have $f_{0}=-\psi^{\prime}(0) / \psi(0)=0$ for the even states and choose $f_{0}=0$ for the odd ones in order to remove the unbalanced term $2 f_{0} / x$ that appears when we substitute the expansion (7) into the Riccati equation (6). Note that this application of the RPM to even potentials is different from the one in earlier papers [2-4] because in the present case we restrict the calculation to the half line. The reason for such a modification is that the present even potentials cannot be expanded in an $x^{2}$ power series.

With the coefficients of the expansion (7) we construct the Hankel determinants $H_{D}^{d}=\left|f_{j+j+d-1}\right|_{i, j=1}^{D}$ that are polynomial functions of the energy and obtain the approximate eigenvalues from the roots of $H_{D}^{d}(E)=0$ [2-4]. More precisely, we expect that sequences of roots $E_{n}^{[D, d]}$ converge towards the eigenvalues $E_{n}, n=0,1, \ldots$, of the corresponding Hamiltonian operator as $D$ increases. Since the SUSY partner potentials on the half line $V_{ \pm}(x)=x^{4} \pm 2 x$ satisfy $V_{+}(-x)=V_{-}(x)$ then the RPM yields the eigenvalues of both partner Hamiltonians simultaneously [5]. More precisely, the sequences of roots for the even and odd wavefunctions will converge towards $E_{0}^{-}<E_{0}^{+}<$ $E_{2}^{-}<E_{2}^{+}<\ldots$ and $E_{1}^{-}<E_{1}^{+}<E_{3}^{-}<E_{3}^{+}<\ldots$, respectively.

Table 3 shows the lowest eigenvalues for the SUSY partner Hamiltonians estimated from Hankel sequences with $D \leq 30, d=0$ and $d=1$. The RPM yields the groundstate eigenvalue of $H_{-}$exactly $\left(E_{0}^{-}=0\right)$ and there is no doubt from present accurate eigenvalues that $E_{n}^{-}=E_{n-1}^{+}$. Marques et al. [1] estimated the ground-state eigenvalues for the Schrödinger equation with the potentials $V(x)=x^{4}$ and $V_{+}(x)$ from the exact ground-state solution $\psi_{0}^{-}, E_{0}^{-}$for $H_{-}$. They resorted to logarithmic perturbation theory but their results were not accurate. Here, we point out that they could have obtained more accurate results by means of the variational method discussed above. In Table 4 we show the results coming from the more efficient RayleighRitz variational method proposed in this paper and we also add the accurate eigenvalues produced by the RPM for comparison.

\section{Conclusions}

It is well known that the Rayleigh-Ritz variational method is more efficient if one treats every symmetry species separately. In the present case we have carried out the separation into even and odd functions on the half line by means 
of appropriate boundary conditions at origin. Thus, the size of present secular equations, and consequently the computational effort for a given accuracy, is one half that of Marques et al. [1].

Working on the half line and taking into account the boundary conditions at origin also enables one to obtain highly accurate eigenvalues by means of the RPM. In the present case one has to be cautious because the same Hankel determinant exhibits roots close to the eigenvalues of both partner Hamiltonian operators. However, it is not difficult to identify the sequence of roots that converges to a chosen eigenvalue. The present RPM results confirm that the two Hamiltonian operators proposed by Marques et al. [1] are already SUSY partners.

The RPM was applied to sequences of SUSY partner potentials in the past [6]. However, the main interest in that paper was to obtain excited-state eigenvalues with Hankel determinants of relatively small dimension. Besides, those potentials can be expanded in $x^{2}$-power series and can therefore be treated in the usual way.

\section{References}

[1] F. Marques, O. Negrini, A. J. da Silva, J. Phys. A 45, 115307 (2012)

[2] F. M. Fernández, Q. Ma, R. H. Tipping, Phys. Rev. A 39, 1605 (1989)

[3] F. M. Fernández, Q. Ma, R. H. Tipping, Phys. Rev. A 40, 6149 (1989)

[4] F. M. Fernández, arXiv:0807.0655v2 [math-ph]

[5] F. M. Fernández, J. Phys. A 29, 3167 (1996)

[6] F. M. Fernández, Q. Ma, D. J. DeSmet, R. H. Tipping, Can. J. Phys. 67, 931 (1989) 